\title{
Condicionamento fisiológico de sementes de couve-flor e desempenho das plantas em campo
}

\author{
Julio Marcos Filho ${ }^{1 ; 3}$; Ana Lúcia P Kikuti ${ }^{2 ; 4}$ \\ ${ }^{1}$ USP/ESALQ, Depto. Produção Vegetal, C. Postal 09, 13418-900 Piracicaba-SP; ${ }^{2}$ Pós-graduanda, USP/ESALQ, fitotecnia. ${ }^{3}$ Bolsista \\ CNPq; ${ }^{4}$ Bolsista FAPESP; jmarcos@esalq.usp.br; alkikuti@esalq.usp.br
}

\section{RESUMO}

O condicionamento fisiológico compreende técnicas que estimulam a germinação e contribuem para reduzir a sensibilidade de sementes e de plântulas a estresses. Efeitos sobre a germinação e a emergência de plântulas têm sido amplamente estudados, mas a influência sobre o comportamento das plantas em campo ainda não é totalmente conhecida. Esta pesquisa foi conduzida com o objetivo principal de verificar efeitos do hidrocondicionamento sobre a germinação, emergência de plântulas, desenvolvimento das plantas e produção de couve-flor. Sementes das cultivares de couve-flor Sharon e Teresópolis Gigante, representados por três lotes, foram hidrocondicionados em papel-toalha, a $20^{\circ} \mathrm{C}$, até atingir $38 \%$ de água ('Sharon') ou 41\% ('Teresópolis Gigante'). Posteriormente, foram secadas em estufa, a $28-30^{\circ} \mathrm{C}$ e $45-55 \%$ de umidade relativa, até atingirem teores de água de 7,5\% a 7,9\%. O potencial fisiológico das sementes foi determinado em laboratório, avaliando-se a percentagem e a velocidade de germinação. Posteriormente, efetuou-se a semeadura em bandejas de poliestireno; o transplante para o campo foi efetuado quando as plantas atingiram estádio de quatro folhas definitivas (30 dias após a semeadura). Determinou-se a velocidade e percentagem de emergência das plântulas aos 14 dias após a semeadura; a altura e a massa seca das plantas foram avaliadas aos 14; 30; 56; 74 e 84 dias e o diâmetro médio da cabeça e a produção final, aos 110 dias. O hidrocondicionamento pode favorecer a velocidade de germinação e de emergência de plântulas, mas esses efeitos não são suficientes para persistir durante o desenvolvimento das plantas e afetar a produção final.

Palavras-chave: Brassica oleracea var. botrytis, hidrocondicionamento, germinação, vigor, produção.

\begin{abstract}
Hydropriming seed treatment and plant field performance

Priming treatment usually promotes rapid and uniform germination and increases tolerance to different stress conditions. Post-treatment effects on seed germination and seedling emergence have been extensively studied but information of plant performance is less available. This research was conducted to verify the influence of hydropriming on seedling emergence, plant development and yield of cauliflower. Sharon and Teresópolis Gigante cultivars, each one represented through three seed lots were primed between two moistened germination paper towel layers under $20^{\circ} \mathrm{C}$. Seeds from 'Sharon' attained 38\% of moisture and, 'Teresópolis Gigante', $41 \%$ of moisture. After the conditioning treatment, seeds were dried to $7.5 \%-7.9 \%$ of moisture in oven with air circulation, at $28-30^{\circ} \mathrm{C}$ and $45-55 \%$ R.H. Seed physiological potential was evaluated in laboratory by speed and percentage of germination. Seeds of each lot were planted in multicell polystyrene trays (200 cells/tray) containing artificial growing mix, and seedlings were transplanted into a field plot when they reached the four-leave stage (30 days after sowing). Seedling emergence (percentage and speed) was determined 14 days after sowing date, and plant height and plant dry weight were measured at $14 ; 30 ; 44 ; 56$, and 74 days. Head diameter and yield were recorded at 110 days. Hydropriming treatment promoted increases in speed of germination and in percentage and speed of seedling emergence, but those effects were not enough to persist during plant growth, not affecting the cauliflower yield.
\end{abstract}

Keywords: Brassica oleracea var. botrytis, seed enhancement, germination, vigor, yield.

(Recebido em 27 de novembro de 2006; aceito em 11 de abril de 2008)

\begin{abstract}
A semente atinge o máximo potencial fisiológico por ocasião da maturidade e, a partir desse ponto, podem ocorrer os primeiros eventos do processo de deterioração. Esses fatos não são colocados em dúvida pela comunidade científica, mas há divergências quanto a possibilidade de reversão dos efeitos da deterioração. É pouco provável que cada semente componente de um lote possa ser regenerada definitivamente com o auxílio de qualquer técnica especial, pois a atuação de mecanismos de reparo implica em consumo de energia e esta não é renovável (Marcos Filho, 2005).
\end{abstract}

Nesse cenário, a pesquisa tem procurado desenvolver procedimentos para uniformizar o desempenho de lotes de sementes ou realçar componentes específicos da qualidade. Esses tratamentos incluem o condicionamento fisiológico (Taylor et al., 1998), cujos benefícios mais evidentes incluem o estímulo à velocidade de germinação ou de emergência de plântulas e a sincronização e uniformidade desses processos; há vantagens ao estabelecimento do estande, particularmente quanto à competição com plantas invasoras e à redução da exposição das plântulas a condições menos favoráveis de ambiente (Nascimento, 1998). Várias pesquisas têm demonstrado essa possibilidade, mas geralmente os estudos concentram-se na identificação de efeitos sobre a germinação e desenvolvimento inicial das plantas.

O condicionamento fisiológico é uma técnica que envolve o controle da hidratação das sementes, de maneira suficiente para ativar os processos metabólicos essenciais para a germinação, mas evitando a protrusão da raiz primária (Heydecker et al., 1975). Sua eficiência foi avaliada em sementes de várias espécies, dentre as quais hortaliças como alface (Eira \& Marcos Filho, 1990), milho-doce (Parera \& Cantliffe, 1994), brócolos (Jett et al., 1996), berinjela (Trigo \& Trigo, 1999), cenoura (Carneiro et al., 1999), pimentão (Roveri José 
et al., 2000) e cebola (Caseiro et al., 2004).

Dentre os procedimentos disponíveis, destacam-se o hidrocondicionamento, o osmocondicionamento e o matricondicionamento (Bradford, 1986; Taylor et al., 1998). O condicionamento de sementes de brássicas não tem recebido atenção significativa da pesquisa. Por exemplo, Rao et al. (1987) verificaram benefícios à emergência de plântulas de couve-de-bruxelas e de couve comum. Em sementes de couve-flor, Fujikura et al. (1993) destacaram efeitos benéficos do condicionamento osmótico sobre a velocidade de germinação, enquanto Thornton \& Powell (1995) relataram vantagens quanto à velocidade de germinação e à tolerância às condições do teste de deterioração controlada, após o hidrocondicionamento.

Após o condicionamento, as sementes atingem teores de água relativamente elevados e inadequados para a conservação do potencial fisiológico durante o armazenamento. Desta maneira, a secagem deve ser conduzida de maneira adequada, para minimizar a possibilidade de reversão dos efeitos benéficos do tratamento.

Vários autores relataram reversão dos efeitos benéficos do tratamento (Fessel et al., 2002), mas outros como Barbedo et al. (1997) e Caseiro (2003) destacaram que o comportamento das sementes depende do genótipo, dos métodos utilizados para o tratamento e das condições de armazenamento. De acordo com Bruggink et al. (1999), a secagem rápida permite a redução segura do grau de umidade, sem provocar a reversão.

Por outro lado, informações sobre o efeito do condicionamento no desenvolvimento das plantas e na produção final da cultura ainda são escassas. Em melão, Nascimento \& West (1999) comentaram que o condicionamento osmótico não influenciou o desenvolvimento das plântulas, mas Evans \& Pill (1989), trabalhando com aspargo, verificaram que esse tratamento beneficiou o estabelecimento inicial das plantas em campo, enquanto Rossetto et al. (1998) destacaram efeitos positivos do condicionamento sobre a pro- dutividade de beterraba. De um modo geral, havendo diferenças no estande inicial, a produtividade pode ser afetada; porém, segundo Larsen et al. (1998), a influência do vigor sobre o desempenho das sementes no campo pode existir mesmo quando não há diferenças no estande inicial.

Desta maneira, as informações sobre efeitos do condicionamento fisiológico de sementes de couve-flor são insuficientes, o mesmo ocorrendo com relação ao desenvolvimento e produção final das plantas provenientes de sementes condicionadas. Essas considerações justificaram a realização da presente pesquisa.

\section{MATERIAL E MÉTODOS}

O trabalho foi conduzido no Laboratório de Análise de Sementes e em campo experimental da ESALQ, de janeiro a outubro de 2005. Na primeira etapa, as sementes foram hidrocondicionadas e secadas. Em seguida, efetuaram-se observações para avaliar o comportamento das sementes e das plantas em campo, comparadas à testemunha não condicionada.

Para tanto, amostras de três lotes de sementes de duas cultivares de couveflor, 'Sharon' e 'Teresópolis Gigante', foram embebidas entre duas camadas de folhas de papel toalha, com quantidade de água equivalente a 2,5 vezes o peso do substrato, a $20^{\circ} \mathrm{C}$, até atingirem teor de água de 38\% ('Sharon', após 30-36 horas) ou de $41 \%$ ('Teresópolis Gigante', após 36 horas).

Em seguida, efetuou-se a secagem das sementes em estufa, a $28-30^{\circ} \mathrm{C}$ e $45-$ $55 \%$ de umidade relativa do ar, durante 20 horas, até atingirem teores de água próximos ao inicial $(7,5-7,9 \%)$. Parte das amostras não foi secada, enquanto as sementes não condicionadas permaneceram com, aproximadamente, 6,0$6,2 \%$ de água.

$\mathrm{Na}$ avaliação em campo, o teste de germinação foi conduzido com quatro repetições de 50 sementes para cada lote e cultivar, distribuídas sobre papel, em caixas plásticas de $11 \times 11 \times 3 \mathrm{~cm}$ a $25^{\circ} \mathrm{C}$, computando-se a percentagem de plântulas normais aos 10 dias após semeadura, conforme as Regras para
Análise de Sementes (Brasil, 1992). Nesse mesmo teste, mediante cômputo diário das plântulas normais, calcularam-se índices médios de velocidade de germinação para cada lote e cultivar (Maguire, 1962). Os tratamentos (sementes não condicionadas, submetidas ao condicionamento fisiológico e ao condicionamento fisiológico seguido por secagem) foram distribuídos segundo delineamento inteiramente casualizado, em arranjo fatorial.

Após as avaliações em laboratório, efetuou-se a semeadura em bandejas de poliestireno contendo substrato comercial (Plantmax para couve-flor), com quatro repetições de 50 sementes por tratamento. A percentagem de emergência das plântulas foi avaliada aos 14 dias após a semeadura, enquanto a velocidade foi computada conforme já descrito, considerando-se as plântulas com tamanho mínimo de $1,0 \mathrm{~cm}$.

Quando as plantas de cada lote atingiram estádio de quatro folhas definitivas, aos 30 dias após a semeadura, efetuou-se o transplante para canteiro, segundo delineamento blocos ao acaso. Adotou-se espaçamento de 0,6 m entre fileiras e de 0,7 m entre covas de plantas. A adubação (na época de semeadura, em cobertura e foliar), irrigação e tratos culturais foram realizados conforme Filgueira (2002).

Os efeitos dos tratamentos foram avaliados mediante determinações da massa seca e altura de plantas aos 14; 30; 56; 74 e 84 dias após a semeadura; a altura foi avaliada em 20 plantas/parcela e a massa seca, em 10 plantas/parcela. Durante a colheita foram consideradas plantas com cabeça completamente desenvolvida, compacta e com botões florais ainda unidos; determinou-se a produção por parcela, posteriormente corrigida para t/ha.

a) Altura das plantas: foram tomadas medidas a partir do colo da planta até o meristema apical, em centímetros, sendo os resultados médios expressos em $\mathrm{cm} /$ planta.

b) Massa seca: para essa determinação, as plantas foram colocadas em sacos de papel e mantidas em estufa com circulação de ar, a $70^{\circ} \mathrm{C}$, até atingirem peso constante. $\mathrm{O}$ material seco foi pesado com precisão de 0,001 g, obtendose a massa seca média (g/planta). 
Tabela 1. Valores médios e coeficientes de variação (\%) referentes à percentagem de germinação (G) e de emergência de plântulas (EP), velocidade de germinação (VG) e de emergência de plântulas (VE) provenientes de três lotes de sementes de couve-flor 'Sharon' e três de 'Teresópolis Gigante' submetidos a hidrocondicionamento seguido ou não por secagem. Piracicaba, USP/ESALQ, 2005.

\begin{tabular}{|c|c|c|c|c|c|c|c|c|c|c|c|c|c|c|}
\hline \multirow{6}{*}{ Sharon } & \multirow[t]{2}{*}{ Teste } & \multicolumn{4}{|c|}{ Testemunha } & \multicolumn{4}{|c|}{ Hidrocondicionamento } & \multicolumn{4}{|c|}{$\begin{array}{c}\text { Hidrocondicionamento + } \\
\text { Secagem }\end{array}$} & \multirow{2}{*}{$\begin{array}{l}\text { CV } \\
(\%)\end{array}$} \\
\hline & & L1 & L2 & L3 & Média & L1 & L2 & L3 & Média & L1 & L2 & L3 & Média & \\
\hline & $\overline{G(\%)}$ & $97 a$ & $100 \mathrm{a}$ & $98 a$ & $98 \mathrm{~A}$ & $98 a$ & $99 a$ & $99 a$ & $99 \mathrm{~A}$ & $97 a$ & $100 \mathrm{a}$ & $98 a$ & $98 \mathrm{~A}$ & 2,85 \\
\hline & VG (índice) & $15,0 \mathrm{~b}$ & 16,3 a & $15,3 \mathrm{~b}$ & $15,5 \mathrm{~B}$ & $15,9 \mathrm{~b}$ & $17,7 \mathrm{a}$ & $16,3 \mathrm{~b}$ & $16,6 \mathrm{~A}$ & 15,7 a & $16,1 \mathrm{a}$ & $15,7 \mathrm{a}$ & $15,8 \mathrm{~B}$ & 3,53 \\
\hline & VE (índice) & $12,5 \mathrm{a}$ & $12,4 \mathrm{a}$ & $11,8 \mathrm{a}$ & $12,2 \mathrm{~B}$ & $16,0 \mathrm{a}$ & $16,6 \mathrm{a}$ & $16,1 \mathrm{a}$ & $16,2 \mathrm{~A}$ & $15,5 \mathrm{~b}$ & $16,6 a$ & $16,5 \mathrm{a}$ & $16,2 \mathrm{~A}$ & 3,42 \\
\hline & $\mathrm{EP}(\%)$ & $100 a$ & $100 \mathrm{a}$ & $97 \mathrm{a}$ & $99 \mathrm{~A}$ & $99 \mathrm{a}$ & $100 \mathrm{a}$ & $100 \mathrm{a}$ & $100 \mathrm{~A}$ & $95 \mathrm{~b}$ & $100 \mathrm{a}$ & $100 \mathrm{a}$ & $98 \mathrm{~A}$ & 2,67 \\
\hline \multirow{6}{*}{$\begin{array}{l}\text { Teresópolis } \\
\text { Gigante }\end{array}$} & \multirow[t]{2}{*}{ Teste } & \multicolumn{4}{|c|}{ Testemunha } & \multicolumn{4}{|c|}{ Hidrocondicionamento } & \multicolumn{4}{|c|}{$\begin{array}{c}\text { Hidrocondicionamento + } \\
\text { Secagem }\end{array}$} & \multirow{2}{*}{$\begin{array}{l}\text { CV } \\
(\%)\end{array}$} \\
\hline & & L4 & L5 & L6 & Média & L4 & L5 & L6 & Média & L4 & L5 & L6 & Média & \\
\hline & $\overline{G(\%)}$ & $95 a$ & $99 a$ & $93 a$ & $96 \mathrm{~A}$ & $95 a$ & $98 a$ & $95 a$ & $96 \mathrm{~A}$ & $96 a$ & $100 a$ & $93 a$ & $96 \mathrm{~A}$ & 4,14 \\
\hline & VG (índice) & $13,1 \mathrm{a}$ & $14,0 \mathrm{a}$ & $13,4 \mathrm{a}$ & $13,5 \mathrm{C}$ & $18,4 a b$ & $19,2 \mathrm{a}$ & $17,3 \mathrm{~b}$ & $18,3 \mathrm{~A}$ & $15,1 \mathrm{~b}$ & $16,1 \mathrm{a}$ & $14,7 \mathrm{~b}$ & $15,3 \mathrm{~B}$ & 4,61 \\
\hline & VE (índice) & $10,8 a$ & $10,7 \mathrm{a}$ & $10,5 \mathrm{a}$ & $13,8 \mathrm{~B}$ & $14,7 \mathrm{a}$ & $15,1 \mathrm{a}$ & $14,2 \mathrm{a}$ & $14,7 \mathrm{~A}$ & $13,5 b$ & $15,1 \mathrm{a}$ & $13,7 \mathrm{~b}$ & $14,1 \mathrm{AB}$ & 6,37 \\
\hline & EP $(\%)$ & $90 \mathrm{a}$ & $91 \mathrm{a}$ & $87 a$ & $89 \mathrm{~B}$ & $93 a$ & $97 \mathrm{a}$ & $92 \mathrm{a}$ & $94 \mathrm{~A}$ & $89 a$ & 96 a & $93 a$ & $93 \mathrm{~A}$ & 6,26 \\
\hline
\end{tabular}

Letras minúsculas: comparações entre médias de lotes; letras maiúsculas: comparação entre tratamentos dentro de cada lote (teste de Tukey, $5 \%$ de probabilidade)

c) Produção: a colheita foi efetuada 78 dias após o transplante das mudas (108 dias após a semeadura), coletando-se quatro plantas/parcela e efetuando-se as determinações: (1) diâmetro da cabeça (medição da distância entre suas extremidades em cm); (2) produção (pesagem das cabeças, calculando-se o peso médio por lote, posteriormente ajustado para t/ha).

A análise estatística foi efetuada separadamente para cada parâmetro avaliado e cultivar, determinando-se os efeitos de tratamentos e de lotes, em delineamento blocos casualizados. As médias foram comparadas pelo método de Tukey (5\% de probabilidade).

\section{RESULTADOS E DISCUSSÃO}

Os lotes de sementes das duas cultivares apresentavam germinação acentuadamente superior ao mínimo (>80\%) estabelecido para a comercialização de sementes de couve-flor (Tabela 1). Em geral, não deve ser esperada ampla resposta de sementes de alto potencial fisiológico ao condicionamento, considerando que os principais objetivos desse tratamento são a aceleração e a sincronização da germinação, normalmente verificadas em lotes de germinação elevada (Marcos Filho, 2005).

No entanto, como estava prevista a secagem pós-condicionamento, que pode provocar reversão dos efeitos do tratamento, optou-se pelo uso de lotes com germinação aceitável pelo comércio. Os resultados obtidos demonstraram que os testes utilizados detectaram diferenças entre os lotes, indicando que sua escolha foi adequada para avaliar o potencial fisiológico das sementes.

$\mathrm{O}$ condicionamento fisiológico e secagem não afetaram significativamente a percentagem de germinação das sementes de 'Sharon', conforme esperado (Tabela 1). Na literatura tem sido ressaltado com grande freqüência, que o condicionamento fisiológico geralmente não promove alteração da percentagem de germinação (Marcos Filho, 2005). Verificaram-se, porém, efeitos dos tratamentos sobre a velocidade de germinação das sementes das duas cultivares e a percentagem e velocidade de emergência de plântulas de 'Teresópólis Gigante' (Tabela 1). Esses resultados confirmaram as observações efetuadas por Fujikura et al. (1993) e por Thorton \& Powell (1995), com sementes de couve-flor.

As determinações destacadas indicaram desempenho superior das sementes tratadas, secadas ou não, em relação à testemunha. Essas informações são importantes porque indicam não só possíveis vantagens do condicionamento fisiológico sobre o estabelecimento das plântulas, como a ausência de reversão desses efeitos com a secagem das sementes. A secagem rápida, na ausência de diferenças com relação a outros métodos considerados eficientes, é preferível devido à maior facilidade de execução. Barbedo et al. (1997), Caseiro (2003) e Bruggink et al. (1999), observaram que a secagem rápida permite a manutenção dos benefícios do condicionamento fisiológico.

Por outro lado, avaliações da velocidade de germinação e de emergência de plântulas também detectaram diferenças, mesmo ao serem comparados lotes com germinação superior a 90\%. Desta maneira, o lote 1 de 'Sharon' e o lote 6 de 'Teresópolis Gigante', geralmente acompanhado pelos lotes 3 e 4, apresentaram desempenho inferior, enquanto os lotes 2 e 5, respectivamente, destacaram-se como superiores (Tabela 1).

Na Tabela 2 são apresentados os resultados referentes ao desempenho de plantas de 'Sharon' provenientes de sementes submetidas ao condicionamento e à secagem, quando comparados à testemunha. Constatou-se ausência de efeitos consistentes dos tratamentos sobre a altura e a massa seca das plantas ao longo do desenvolvimento, embora em alguns casos excepcionais, como para a altura das plantas aos 14 e aos 74 dias após a semeadura e para massa seca aos 74 dias, tenham ocorrido diferenças significativas entre tratamento(s) e a testemunha. Esses, no entanto, foram considerados efeitos esporádicos que não caracterizaram forte tendência, pois aos 
Tabela 2. Valores médios e coeficientes de variação (\%) referentes à altura e massa seca de plantas, em diferentes estádios de desenvolvimento, diâmetro e produção de cabeças de couve-flor cv. Sharon, a partir de três lotes de sementes submetidos ao hidrocondicionamento seguido ou não por secagem. Piracicaba, USP/ESALQ, 2005.

\begin{tabular}{|c|c|c|c|c|c|c|c|c|c|c|c|}
\hline \multirow{2}{*}{ Lotes } & \multirow{2}{*}{ Tratamentos } & \multicolumn{4}{|c|}{ Altura (cm) } & \multicolumn{4}{|c|}{ Massa seca $(g)$} & \multirow{2}{*}{$\begin{array}{c}\text { Diâmetro } \\
\text { cabeça } \\
(\mathrm{cm})\end{array}$} & \multirow{2}{*}{$\begin{array}{c}\text { Produção } \\
\text { (t/ha) }\end{array}$} \\
\hline & & $14 \mathrm{~d}$ & $30 \mathrm{~d}$ & $56 \mathrm{~d}$ & $74 \mathrm{~d}$ & $14 \mathrm{~d}$ & $30 \mathrm{~d}$ & $56 \mathrm{~d}$ & $74 \mathrm{~d}$ & & \\
\hline \multirow{4}{*}{ Lote 1} & Testemunha & $2,86 \mathrm{~A}$ & $5,85 \mathrm{~A}$ & $5,62 \mathrm{~A}$ & $13,10 \mathrm{~A}$ & $0,022 \mathrm{~A}$ & $0,141 \mathrm{~A}$ & $5,794 \mathrm{~A}$ & $47,583 \mathrm{~A}$ & $23,75 \mathrm{~A}$ & $16,3 \mathrm{~A}$ \\
\hline & Hidrocond. & $2,75 \mathrm{~A}$ & $5,14 \mathrm{~A}$ & $5,37 \mathrm{~A}$ & $11,88 \mathrm{~B}$ & $0,025 \mathrm{~A}$ & $0,126 \mathrm{~A}$ & $5,609 \mathrm{~A}$ & $43,972 \mathrm{~A}$ & $22,04 \mathrm{~A}$ & $14,9 \mathrm{~A}$ \\
\hline & Hidroc. + Sec. & $2,67 \mathrm{~A}$ & $5,07 \mathrm{~A}$ & $5,23 \mathrm{~A}$ & $12,42 \mathrm{AB}$ & $0,023 \mathrm{~A}$ & $0,130 \mathrm{~A}$ & $6,191 \mathrm{~A}$ & $45,320 \mathrm{~A}$ & $22,00 \mathrm{~A}$ & $15,6 \mathrm{~A}$ \\
\hline & Média & $2,76 \mathrm{~b}$ & $5,35 \mathrm{~b}$ & $5,41 \mathrm{a}$ & $12,50 \mathrm{~b}$ & $0,023 \mathrm{~b}$ & $0,132 \mathrm{~b}$ & 5,865 a & $45,625 a b$ & $22,60 \mathrm{a}$ & $15,6 \mathrm{a}$ \\
\hline \multirow{4}{*}{ Lote 2} & Testemunha & $3,43 \mathrm{~A}$ & $6,12 \mathrm{~A}$ & $5,32 \mathrm{~A}$ & $14,17 \mathrm{~A}$ & $0,044 \mathrm{~A}$ & $0,198 \mathrm{~A}$ & $6,633 \mathrm{~A}$ & $54,070 \mathrm{~A}$ & $22,25 \mathrm{~A}$ & $15,4 \mathrm{~A}$ \\
\hline & Hidrocond. & $3,65 \mathrm{~A}$ & $6,28 \mathrm{~A}$ & $5,58 \mathrm{~A}$ & $13,25 \mathrm{~B}$ & $0,040 \mathrm{~A}$ & $0,164 \mathrm{~A}$ & $6,376 \mathrm{~A}$ & $49,377 \mathrm{~B}$ & $22,38 \mathrm{~A}$ & $14,6 \mathrm{~A}$ \\
\hline & Hidroc. + Sec. & $3,52 \mathrm{~A}$ & $5,92 \mathrm{~A}$ & $5,82 \mathrm{~A}$ & $13,00 \mathrm{~B}$ & $0,030 \mathrm{~B}$ & $0,128 \mathrm{~A}$ & $6,046 \mathrm{~A}$ & $48,492 \mathrm{~B}$ & $22,33 \mathrm{~A}$ & $14,8 \mathrm{~A}$ \\
\hline & Média & $2,96 \mathrm{~b}$ & $6,11 \mathrm{a}$ & 5,57 a & 13,47 a & $0,038 a$ & $0,163 a$ & $6,352 \mathrm{a}$ & 50,646 a & $22,32 \mathrm{a}$ & 14,9 a \\
\hline \multirow{4}{*}{ Lote 3} & Testemunha & $3,05 \mathrm{~A}$ & $6,54 \mathrm{~A}$ & $6,45 \mathrm{~A}$ & $12,92 \mathrm{~A}$ & $0,028 \mathrm{~A}$ & $0,180 \mathrm{~A}$ & $6,389 \mathrm{~A}$ & $46,900 \mathrm{~A}$ & $24,71 \mathrm{~A}$ & $15,8 \mathrm{~A}$ \\
\hline & Hidrocond. & $2,84 \mathrm{~A}$ & $6,26 \mathrm{~A}$ & $6,40 \mathrm{~A}$ & $12,55 \mathrm{~A}$ & $0,029 \mathrm{~A}$ & $0,123 \mathrm{~A}$ & $6,685 \mathrm{~A}$ & $41,063 \mathrm{~B}$ & $23,50 \mathrm{~A}$ & $15,3 \mathrm{~A}$ \\
\hline & Hidroc. + Sec. & $3,00 \mathrm{~A}$ & $6,46 \mathrm{~A}$ & $6,60 \mathrm{~A}$ & $12,15 \mathrm{~A}$ & $0,030 \mathrm{~A}$ & $0,126 \mathrm{~A}$ & $6,546 \mathrm{~A}$ & $43,840 \mathrm{AB}$ & $22,69 \mathrm{~A}$ & $15,1 \mathrm{~A}$ \\
\hline & Média & $3,53 \mathrm{a}$ & $5,75 a b$ & $6,48 \mathrm{a}$ & $12,54 \mathrm{~b}$ & $0,029 a b$ & $0,143 a b$ & $6,540 \mathrm{a}$ & $43,934 \mathrm{~b}$ & $23,63 a$ & $15,4 \mathrm{a}$ \\
\hline$\overline{C V}(\%)$ & & 10,64 & 9,38 & 17,46 & 9,05 & 12,18 & 18,82 & 28,59 & 27,89 & 6,41 & 11,77 \\
\hline
\end{tabular}

Letras minúsculas: comparações entre médias de lotes; letras maiúsculas: comparação entre tratamentos dentro de cada lote (teste de Tukey, $5 \%$ de probabilidade)

Tabela 3. Valores médios e coeficientes de variação (\%) referentes à altura e massa seca de plantas, em diferentes estádios de desenvolvimento, diâmetro e produção de cabeças de couve-flor cv. Teresópolis Gigante, a partir de três lotes de sementes submetidos ao hidrocondicionamento seguido ou não por secagem. Piracicaba, USP/ESALQ, 2005.

\begin{tabular}{|c|c|c|c|c|c|c|c|c|c|c|c|}
\hline \multirow{2}{*}{ Lotes } & \multirow{2}{*}{ Tratamentos } & \multicolumn{4}{|c|}{ Altura $(\mathrm{cm})$} & \multicolumn{4}{|c|}{ Massa seca $(g)$} & \multirow{2}{*}{$\begin{array}{c}\text { Diâmetro } \\
\text { cabeça } \\
(\mathrm{cm})\end{array}$} & \multirow{2}{*}{$\begin{array}{l}\text { Produção } \\
\text { (t/ha) }\end{array}$} \\
\hline & & $14 d$ & $30 \mathrm{~d}$ & $56 \mathrm{~d}$ & $74 d$ & $14 \mathrm{~d}$ & $30 \mathrm{~d}$ & $56 \mathrm{~d}$ & $74 \mathrm{~d}$ & & \\
\hline \multirow{4}{*}{ Lote 4} & Testemunha & $2,59 \mathrm{~A}$ & $3,66 \mathrm{~A}$ & $3,82 \mathrm{~A}$ & $8,70 \mathrm{~A}$ & $0,016 \mathrm{~A}$ & $0,121 \mathrm{~A}$ & $4,872 \mathrm{~A}$ & $42,151 \mathrm{AB}$ & $21,50 \mathrm{AB}$ & $14,9 \mathrm{~A}$ \\
\hline & Hidroc. & $2,35 \mathrm{~A}$ & $3,54 \mathrm{~A}$ & $3,23 \mathrm{~A}$ & $9,18 \mathrm{~A}$ & $0,021 \mathrm{~A}$ & $0,094 \mathrm{~A}$ & $4,683 \mathrm{~A}$ & $47,053 \mathrm{~A}$ & $23,17 \mathrm{~A}$ & $13,7 \mathrm{~A}$ \\
\hline & Hidroc. + Sec. & $2,32 \mathrm{~A}$ & $3,20 \mathrm{~A}$ & $4,27 \mathrm{~A}$ & $7,88 \mathrm{~B}$ & $0,020 \mathrm{~A}$ & $0,080 \mathrm{~A}$ & 3,837 B & $38,008 \mathrm{~B}$ & $19,96 \mathrm{~B}$ & $13,9 \mathrm{~A}$ \\
\hline & Média & $2,42 a$ & $3,46 a b$ & $3,77 \mathrm{a}$ & $8,59 a$ & $0,019 a$ & $0,098 \mathrm{~b}$ & $4,464 a b$ & $42,40 \mathrm{a}$ & $21,54 \mathrm{a}$ & $14,2 \mathrm{a}$ \\
\hline \multirow{4}{*}{ Lote 5} & Testemunha & $2,35 \mathrm{~A}$ & $3,65 \mathrm{~A}$ & $3,38 \mathrm{~B}$ & $7,33 \mathrm{~A}$ & $0,015 \mathrm{~A}$ & $0,116 \mathrm{~A}$ & $2,867 \mathrm{~B}$ & $36,022 \mathrm{~A}$ & $22,17 \mathrm{~A}$ & $14,9 \mathrm{~A}$ \\
\hline & Hidroc. & $2,41 \mathrm{~A}$ & $3,91 \mathrm{~A}$ & $4,43 \mathrm{~A}$ & $8,03 \mathrm{~A}$ & $0,018 \mathrm{~A}$ & $0,123 \mathrm{~A}$ & $2,802 \mathrm{~B}$ & $40,444 \mathrm{~A}$ & $22,83 \mathrm{~A}$ & $15,1 \mathrm{~A}$ \\
\hline & Hidroc. + Sec. & $2,40 \mathrm{~A}$ & $3,41 \mathrm{~A}$ & $3,97 \mathrm{AB}$ & $8,18 \mathrm{~A}$ & $0,019 \mathrm{~A}$ & $0,123 \mathrm{~A}$ & $5,026 \mathrm{~A}$ & $37,565 \mathrm{~A}$ & $21,83 \mathrm{~A}$ & $14,3 \mathrm{~A}$ \\
\hline & Média & $2,40 \mathrm{a}$ & $3,66 \mathrm{a}$ & $3,96 \mathrm{a}$ & $7,85 \mathrm{a}$ & $0,017 a b$ & $0,121 \mathrm{a}$ & $3,568 \mathrm{~b}$ & 38,014 a & $22,28 \mathrm{a}$ & $15,1 \mathrm{a}$ \\
\hline \multirow{4}{*}{ Lote 6} & Testemunha & $2,55 \mathrm{~A}$ & $3,27 \mathrm{~B}$ & $3,30 \mathrm{~B}$ & $8,85 \mathrm{~A}$ & $0,014 \mathrm{~A}$ & $0,095 \mathrm{~A}$ & $6,612 \mathrm{~A}$ & $39,693 \mathrm{~A}$ & $21,92 \mathrm{~A}$ & $14,5 \mathrm{~A}$ \\
\hline & Hidro. & $2,07 \mathrm{~A}$ & $2,78 \mathrm{~B}$ & $3,40 \mathrm{~B}$ & $7,93 \mathrm{~A}$ & $0,015 \mathrm{~A}$ & $0,105 \mathrm{~A}$ & $3,718 \mathrm{~B}$ & $29,217 \mathrm{~A}$ & $19,29 \mathrm{~A}$ & $13,5 \mathrm{~A}$ \\
\hline & Hidroc. + Sec. & $2,20 \mathrm{~A}$ & $3,87 \mathrm{~A}$ & $4,15 \mathrm{~A}$ & $8,45 \mathrm{~A}$ & $0,017 \mathrm{~A}$ & $0,116 \mathrm{~A}$ & $5,693 \mathrm{~A}$ & $43,319 \mathrm{~A}$ & $20,17 \mathrm{~A}$ & $14,1 \mathrm{~A}$ \\
\hline & Média & $2,27 \mathrm{a}$ & $3,30 \mathrm{~b}$ & $3,62 \mathrm{a}$ & $8,41 \mathrm{a}$ & $0,015 \mathrm{~b}$ & $0,106 a b$ & $5,341 \mathrm{a}$ & $37,409 a$ & $20,46 a$ & $14,0 \mathrm{a}$ \\
\hline$\overline{C V}(\%)$ & & 9,49 & 8,98 & 9,94 & 11,12 & 13,49 & 21,99 & 18,55 & 19,14 & 7,45 & 18,90 \\
\hline
\end{tabular}

Letras minúsculas: comparações entre médias de lotes; letras maiúsculas: comparação entre tratamentos dentro de cada lote (teste de Tukey, $5 \%$ de probabilidade)

84 dias (dados não apresentados) também não ocorreram diferenças entre os tratamentos.

Ao mesmo tempo, a deficiência do desenvolvimento das plantas do lote 1 confirmou os resultados de laboratório, tendo sido constatada inferioridade na altura e na massa seca dos 14 aos 74 dias após a semeadura. Entretanto, não foram detectados efeitos de tratamentos e de lotes sobre a produção das plantas (Tabela 2).
Desta maneira, o condicionamento fisiológico afetou o vigor das sementes, expresso pela velocidade de germinação e de emergência de plântulas. Houve tendências (observadas comparando-se os valores numéricos) desses efeitos refletirem-se sobre o desenvolvimento das plantas. No entanto, não foi detectada influência sobre a produção final, de maneira comparável à enfatizada por Carvalho (1986), TeKrony \& Egli (1991), Ellis (1992), Rodo \& Marcos
Filho (2003), Sengupta et al. (2005), dentre outros.

Observações semelhantes foram efetuadas para 'Teresópolis Gigante'. A expressão do vigor pela velocidade de germinação e de emergência de plântulas, indicou vantagem do hidrocondicionamento, seguido ou não por secagem, e a superioridade do desempenho das sementes do lote 5 (Tabela 1). Esse comportamento do lote 5 também foi constatado nas comparações 
entre os valores numéricos correspondentes aos três lotes avaliados (Tabela 3), principalmente para a altura de plantas entre os 14 e os 56 dias. Entretanto, confirmando os resultados obtidos para a cultivar Sharon, essas diferenças não foram suficientemente amplas para atingir níveis significativos e não se manifestaram quando foram consideradas a massa seca das plantas e a produção de cabeças.

Os resultados obtidos nesta pesquisa indicaram, portanto, que o hidrocondicionamento promove efeitos benéficos sobre a velocidade de germinação e de emergência de plântulas de couve-flor, aspectos diretamente relacionados ao estabelecimento do estande. Essa situação é vantajosa porque acarreta menor período de exposição das sementes a fatores adversos de ambiente após a semeadura. Ao mesmo tempo, o desempenho inicial das plantas também foi beneficiado pelo vigor das sementes, fato destacado para as duas cultivares.

Pode ser constatado, ainda, que os efeitos dos tratamentos e do vigor das sementes não persistiram durante todo o desenvolvimento das plantas, não sendo suficientes para afetar a produção final, em situação onde não houve diferenças acentuadas no estande inicial da cultura. No entanto, os benefícios aqui relatados justificam a utilização de sementes mais vigorosas e/ou submetidas ao hidrocondicionamento, mesmo em lotes com percentagem de germinação elevada, permitindo o estabelecimento rápido e uniforme da cultura, passo importante para a obtenção de rendimentos compensadores.

\section{REFERÊNCIAS}

BARBEDOCJ; MARCOS FILHO J; NOVEMBRE ADLC. 1997. Condicionamento osmótico e armazenamento de sementes de cedro-rosa. Revista Brasileira de Sementes 19: 355-361.

BRADFORD KJ. 1986. Manipulation of seed water relations via osmotic priming to improve germination under stress conditions. HortScience 21: 1105-1112.
BRASIL. Ministério da Agricultura. Departamento Nacional de Produção Vegetal. 1992. Regras para Análise de Sementes. Brasília: SNDA/DNDV/CLAV. 365 p.

BRUGGINK GT; OOMS JJJ; Van Der TOORN P. 1999. Induction of longevity in primed seeds. Seed Science Research 9: 49-53.

CARNEIRO JWP; BRACCINI AL; GUEDES TA; AMARALD. 1999. Influência do estresse hídrico, térmico e do condicionamento osmótico no desempenho germinativo de sementes de cenoura. Revista Brasileira de Sementes 21: 208-216.

CARVALHO NM. 1986. Vigor de sementes. In: MARCOS FILHO J; CICERO SM; SILVA WR. (Eds.) Atualização em produção de sementes. Campinas: Fundação Cargill. p. 207223.

CASEIRO RF. 2003 Métodos para o condicionamento fisiológico de sementes de cebola e influencia da secagem $e$ armazenamento. Piracicaba, USP-ESALQ. 109p. (Tese doutorado).

CASEIRO RF; BENNETT MA; MARCOS FILHO J. 2004. Comparison of three priming techniques for onion seed lots differing in initial seed quality. Seed Science and Technology 32: 365-375.

EIRA MTS; MARCOS FILHO J. 1990. Condicionamento osmótico de sementes de alface: I. Efeitos sobre a germinação. Revista Brasileira de Sementes 1: 9-27.

ELLIS RH. 1992. Seed and seedling vigor in relation to growth and yield. Plant Growth Regulation 11: 249-255.

EVANS TA; PILL WG. 1989. Emergence and seedling growth from osmotically primed or pregerminated seeds of asparagus. Journal of Horticultural Science 64: 275-282.

FESSEL SA; VIEIRA RD; RODRIGUES TJD. 2002. Germinação de sementes de alface submetidas a condicionamento osmótico durante o armazenamento. Scientia Agricola 59: 73-77.

FILGUEIRA FAR. 2002. Novo manual de olericultura: agrotecnologia moderna na produção e comercialização de hortaliças. Viçosa: UFV. 402 p.

FUJIKURA Y; KRAAK HL; BASRA AS; KARSSEN CM. 1993. Hydro priming, a simple and unexpensive priming method. Seed Science and Technology 21: 639-642.

HEYDECKER W; HIGGINS J; TURNER YJ. 1975. Invigoration of seeds? Seed Science and Technology 3: 881-888.

JETT LW; WELBAUM GE; MORSE RD. 1996. Effects of matric and osmotic priming treatments on broccoli seed germination. Journal of American Society of Horticultural Science 121: 423-429.
LARSEN SU; POVLSEN FV; ERIKSEN EN; PEDERSEN HC. 1998. The influence of seed vigour on field performance and the evaluation of the applicability of the controlled deterioration vigour test in oil seed rape and pea. Seed Science and Technology 26: 627-641.

MAGUIRE JD. 1962. Speed of germination-aid in relation evaluation for seedling emergence vigor. Crop Science 2: 176-177.

MARCOS FILHO J. 2005. Fisiologia de sementes de plantas cultivadas. Piracicaba: FEALQ. 495 p.

NASCIMENTO WM. 1998. Condicionamento osmótico de sementes de hortaliças: potencialidades e implicações. Horticultura Brasileira 16: 106-109.

NASCIMENTO WM; WEST SH. 1999. Muskmelon transplant production in response to seed priming. Hort Technology 9: 53-55.

PARERA C; CANTLIFE DJ. 1994. Presowing seed priming. Horticultural Reviews 16: 109139.

RAO SC; AKERS SW; AHRING RM. 1987. Priming brassica seed to improve emergence under different temperatures and soil moisture conditions. Crop Science 27: 1050-1053.

RODO AB; MARCOS FILHO. J. 2003. Onion seed vigor in relation to plant growth and yield. Horticultura Brasileira 21: 220-226.

ROSSETTO CAV; MINAMI K; NAKAGAWA J. 1998. Efeito do condicionamento fisiológico de sementes de beterraba na emergência e na produtividade. Revista Brasileira de Sementes 20: 350-355.

ROVERI JOSÉ SCBR; VIEIRA MGGC; GUIMARÃES RM. 2000. Efeito da temperatura e do período de condicionamento osmótico na germinação e no vigor de sementes de pimentão. Revista Brasileira de Sementes 22: 176-184.

SENGUPTA AK; DE BK; MANDAL AK. 2005. Pré-storage seed invigoration treatments for the maintenance of vigour, viability and field performance of nigh-vigour onion seed. Seed Science and Technology 33: 753-760.

TAYLOR AG; ALLEN PS; BENNETT MA; BRADFORD KJ; BURRIS JS; MISRA MK. 1998. Seed enhancements. Seed Science Research 8: 245-256.

TeKRONY DM; EGLI DB. 1991. Relationship of seed vigor to crop yield. Crop Science 31: 816-822.

THORNTON JM; POWELL AA. 1995. Prolonged aerated hydration for improvement of seed quality in Brassica oleracea L. Annals of Applied Biology 127: 183-189.

TRIGO MFOO; TRIGO LFN. 1999. Efeito do condicionamento osmótico na germinação e no vigor de sementes de berinjela. Revista Brasileira de Sementes 21: 107-113. 